\title{
Guest Editorial: Special Issue on Computational Intelligence for Cloud Computing
}

schemes.

In "Entropy4Cloud: Using Entropy-based Complexity to Optimize Cloud Service Resource Management", Chen et al. identify the origin of complexity in cloud service resource management system through the study of Local Activity Principle. Then they propose an Entropy-based methodology which covers identifying, measuring, analyzing and controlling of complexity. The idea is also implemented in a popular cloud engine, Apache Spark, for running Analysis as a Service (AaaS). Comparisons are given to the Fair Scheduler in Apache Spark. Results show that the proposed Entropy Scheduler has significantly outperformed it.

In "Scheduling for Time-constrained Big-file Transfer over Multiple Paths in Cloud Computing", Lin et al. consider two types of scheduling problems for big-file transfer in cloud computing, ie, single-file transfer scheduling (SFTS) and multifile transfer scheduling (MFTS). Both problems aim to maximize the bandwidth utilization under delay constraint. For SFTS, a heuristic algorithm is developed by adopting maximum flow over time. For MFTS, a heuristic is developed with an intelligent scheme to maximize the throughput and schedule the multi-file flow dynamically.

In "A Deep Learning Approach to Network Intrusion Detection", Shone et al. present a novel deep learning technique for intrusion detection, which addresses the concerns regarding the feasibility and sustainability of current Network Intrusion Detection Systems (NIDSs) approaches. They first propose a non-symmetric deep auto-encoder (NDAE) for unsupervised feature learning and then propose a novel deep learning classification model constructed using stacked NDAEs. The new technique has been implemented in GPU-enabled TensorFlow and evaluated using some popular benchmark datasets. Results demonstrate significant improvements over existing approaches and the strong potential to be applied in modern NIDSs.

Overall, the Special Issue was attractive since it received a total of $\mathbf{x x}$ submissions. The above 4 papers were selected following a very rigorous peer review. The guest editors would like to thank all the authors for their contributions and all the reviewers for their hard work in completing the reviewing timely. Last but not least, we thank Editor-In-Chief, Prof YewSoon Ong, for the constant support and assistance offered during the editing process of this Special Issue.

We hope that you will enjoy reading these novel contributions! 


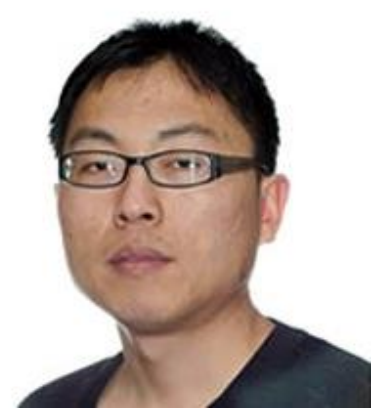

H. Cheng, Guest Editor

Liverpool John Moores University

Liverpool L3 3AF, UK

H.Cheng@ljmu.ac.uk

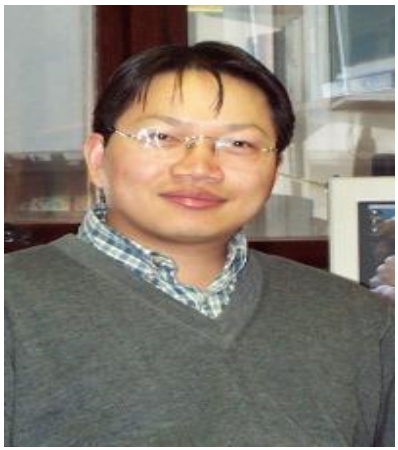

S. Yang, Guest Editor

De Montfort University

Leicester LE1 9BH, UK

syang@dmu.ac.uk.

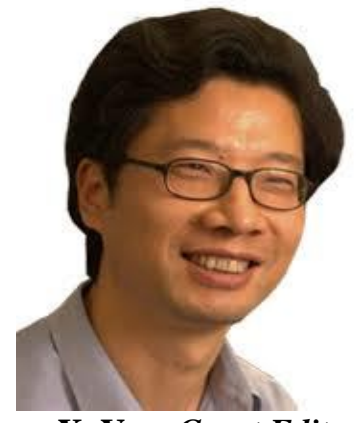

X. Yao, Guest Editor

Southern University of Science and Technology Shenzhen 518055, China xiny@sustc.edu.cn

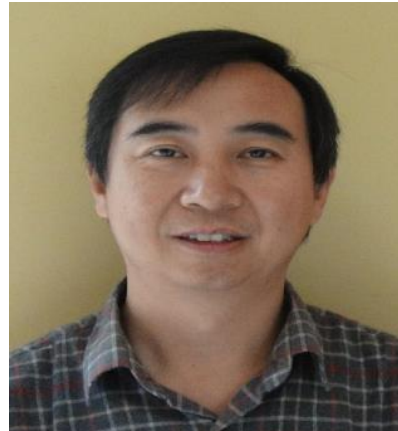

M. ZHANG, Guest Editor Victoria University of Wellington Wellington 6012, New Zealand mengjie.zhang@ecs.vuw.ac.nz 